\title{
Discussing treatment limitations in frail older COVID-19 patients: A Framework Analysis.
}

\author{
Sophie Lochtenberg ${ }^{1}$, Elise $\mathrm{Pel}^{2}$, Roeline Pasman ${ }^{3}$, and Peter Noordzij ${ }^{4}$ \\ ${ }^{1}$ VU University Medical Centre Amsterdam \\ ${ }^{2}$ Erasmus Medical Center \\ ${ }^{3}$ Amsterdam UMC Location AMC \\ ${ }^{4}$ Sint Antonius Ziekenhuis
}

February 15, 2022

\begin{abstract}
Aims and objectives: The COVID-19 pandemic caused an increase in hospitalizations for frail older people and required healthcare professional to make difficult ethical and medical decisions regarding intensive care unit admission and treatment. This study investigates the experiences of healthcare professionals with the use of a decision support tool when discussing treatment limitations with older patients with COVID-19. Methods: A qualitative approach was chosen to obtain further in-depth information on the experiences of the healthcare professionals with the conversations about treatment limitation and on the contribution of a decision support tool for frail older adults with COVID-19. The framework method was used for the data analysis. Results: The following themes illustrate the analyzed concepts for the subject conversations about treatment limitation: careful consideration, the conversation is a part of the job, the burden of the conversation, scheduling conversation and acquiring skills to perform the conversation. The concepts of the theme AGE-ICU evaluation are included in the following themes: considered and comprehensible overview, confirmation of own assessment, every decision is context and person dependent, contributes to considered decision and tool not needed because of own expertise. Conclusion: A decision support tool for older patients with COVID-19 may help the healthcare professional to objectify the patients' health status and functioning and discuss risk factors for adverse outcomes. Besides this, the tool helps to initiate the difficult conversation with the patient and their family. Finally, the AGE-ICU contributes to shared-decision making because it helps patients to understand the suggested decision and patients are more involved in the decision-making process.
\end{abstract}

Discussing treatment limitations in frail older COVID-19 patients: A Framework Analysis.

Sophie Anna Lochtenberg (MS) ${ }^{1 *}$, Elise Linde Pel (MD, MA) ${ }^{*}$, Roeline Pasman ${ }^{1}$, Peter Noordzij (PHD, $\mathrm{MD})^{3}$

*Both authors contributed equally.

1. Department of public and occupational health, Amsterdam Public Health research institute, Expertise Center for Palliative Care, Amsterdam UMC, VU University, Amsterdam, the Netherlands

2. Erasmus Medical Centre, Department of Geriatrics, Rotterdam, The Netherlands

3. St. Antonius Hospital Nieuwegein, Department of Anesthesiology, Intensive Care and pain management, Nieuwegein, The Netherlands.

Corresponding author: E.L. Pel, email address: e.pel@erasmusmc.nl

Abstract 
Aims and objectives: The COVID-19 pandemic caused an increase in hospitalizations for frail older people and required healthcare professional to make difficult ethical and medical decisions regarding intensive care unit admission and treatment. This study investigates the experiences of healthcare professionals with the use of a decision support tool when discussing treatment limitations with older patients with COVID-19.

Methods: A qualitative approach was chosen to obtain further in-depth information on the experiences of the healthcare professionals with the conversations about treatment limitation and on the contribution of a decision support tool for frail older adults with COVID-19. The framework method was used for the data analysis.

Results: The following themes illustrate the analyzed concepts for the subject conversations about treatment limitation: careful consideration, the conversation is a part of the job, the burden of the conversation, scheduling conversation and acquiring skills to perform the conversation. The concepts of the theme AGEICU evaluation are included in the following themes: considered and comprehensible overview, confirmation of own assessment, every decision is context and person dependent, contributes to considered decision and tool not needed because of own expertise.

Conclusion: A decision support tool for older patients with COVID-19 may help the healthcare professional to objectify the patients' health status and functioning and discuss risk factors for adverse outcomes. Besides this, the tool helps to initiate the difficult conversation with the patient and their family. Finally, the AGEICU contributes to shared-decision making because it helps patients to understand the suggested decision and patients are more involved in the decision-making process.

Keywords: treatment limitation, decision-making tool, COVID-19, frailty, framework method.

Introduction

The global pandemic of coronavirus disease 2019 (COVID-19) caused by SARS-CoV-2 has been overwhelming worldwide $^{1}$. According to the World Health Organization in November 2021 more than 260 million people are infected and over 5 million people have died due to COVID-19 globally ${ }^{2,3}$. Consequently, the COVID-19 pandemic caused an increase in hospitalizations for people of all ages with pneumonia ${ }^{1}$. This resulted in shortage of hospital resources and required healthcare professionals to make difficult ethical and medical decisions regarding intensive care unit (ICU) admission and treatment ${ }^{3-5}$.

The decision about ICU admission in frail older patients can be complex and could have serious consequences. Triage criteria are often used to support the healthcare professionals in this decision ${ }^{7}$. Probability of treatment benefit, age and frailty are the most commonly used criteria to admit a person to the ICU $\mathrm{I}^{6,7}$. Frailty increases the risk of negative health outcomes, the chance of complications and is more common in older age $\mathrm{e}^{8,9}$. Older patients with comorbidities have a higher risk of hospitalization and mortality due to COVID-197 . However, ICU admission is not always the right choice for all older patients ${ }^{8}$. It is a burdensome treatment and can have profound short- and long-term consequences ${ }^{6}$. Furthermore, the majority of COVID-19 survivors who are discharged from the ICU often leave with significant morbidity and a long and uncertain road to recovery ${ }^{6}$.

Healthcare professionals always aim to provide care in the best interest of the patients ${ }^{8}$. Usually, only patients with a reasonable chance to recover with a satisfactory health are admitted to the ICU and patients with low chances of survival are not admitted to the ICU ${ }^{10}$. In older frail COVID-19 patients the advantages and disadvantages of ICU treatment need to be carefully weighted. Knowing the complexity and the risks of the treatment, it is important that patients are involved in decision-making ${ }^{11}$. Healthcare professionals are obliged to have a conversation with the patient about the various treatment options including treatment limitations ${ }^{8}$. These conversations can be difficult to initiate and are often avoided by healthcare professionals due to fear of causing distress for the patient ${ }^{12}$. A decision support tool that includes the patients' risks and characteristics can be used to guide healthcare professionals in this conversation and could be the starting point for decision-making. Therefore, risk assessments could be the base for the healthcare professional to inform the patients and their families to make a well-considered decision regarding treatment ${ }^{9}$. Besides this, 
the decision not to be treated in the ICU can prevent patients from complications or dying in solitude due the strict COVID-19 rules that only allows one or two loved ones. Finally, by involving the patients their autonomy is respected ${ }^{13}$.

There is a need for tools to contribute to risk assessment to guide healthcare professionals in decisionmaking during the COVID-19 pandemic ${ }^{11,14}$. Little research has been done on discussing treatment limitations. Berghuis (2019 studied physician-related factors concerning treatment limitations and states that communication training is effective, but the thesis has argued that more insight in perspectives of healthcare professionals is necessary and to find out what facilitates them in these conversations ${ }^{13}$. Griffith et al. (2020) researched the process of decision-making around ICU admission during the COVID-19 pandemic. They concluded that a framework for decision-making around ICU admission in which patients and their families are involved has the potential to improve decision-making and complement clinical prediction tools ${ }^{11}$. Taken together, the reported conclusions of both studies appear to support the assumption that there is a need for a decision support tool that guides healthcare professionals in decision-making with older COVID-19 patients.

The high number of hospitalizations due to COVID-19 caused an increase in conversations about treatment limitation. This demanded a tool to contribute in the risk assessment among older COVID-19 patients. In St. Antonius hospital the Anesthesia Geriatric Evaluation (AGE) screening tool is developed to detect patients with increased frailty prior to high-risk surgery ${ }^{15}$. Based on this tool the AGE-ICU tool is developed during the first COVID-19 wave in March 2020, aiming to support healthcare professionals in the decision about older COVID-19 patients and ICU admission. Examples of aspects that are included in the tool are frailty, and cardiovascular and pulmonary risk factors (Appendix I). The aim of this study was to evaluate the AGE-ICU tool, which is designed to help healthcare professionals with the conversations about treatment limitations in older COVID-19 patients and to investigate the experiences of the healthcare professionals with these conversations.

Method

A qualitative approach was chosen to obtain further in-depth information on the experiences of the healthcare professionals with a decision support tool to discuss treatment limitations in older COVID-19 patients. This tool was used by healthcare professionals at COVID-19 wards in St. Antonius hospital in Nieuwegein, a large teaching hospital in the Netherlands. The tool is called AGE ICU and is described in appendix 1.

Participants

In the period from February till the end of May 2021 participants were recruited from St. Antonius Hospital, Nieuwegein. They were requested to participate by email. It was required that the participants worked with the AGE-ICU tool during the COVID-19 pandemic that started in March 2020. Doctors and residents of all ages, specialties and from various departments were included. Six medical specialists and four residents participated in this research.

Data collection

Semi-structured interviews were conducted with healthcare professionals from St. Antonius hospital who used the AGE-ICU tool during the COVID-19 pandemic. The interviews were based on a predetermined topic guide with example questions (Appendix I). Data collection was applied until no new themes derived from the data, and saturation seemed to be reached.

Analysis

The method chosen for this research is the framework method. This analysis is described by the National Centre for Social Research as a content analysis method which involves summarizing and classifying data within a thematic framework ${ }^{16}$. The framework method consists of seven clear steps to follow and produce highly structured outputs of summarized data ${ }^{17}$. Firstly, the interviews were transcribed verbatim. After transcription the researcher had to get familiar with the data by attentively reading the transcripts again. 
The third step of the framework analysis is coding; the transcripts were analyzed line by line and codes were used for the description of the interpretation of a certain line. The fourth step is the development of a working analytical framework which means that the codes from the third step were grouped into categories. After development of the analytical framework the data was charted into the framework matrix. Lastly, the seventh step considered the interpretation of the data. The researchers have been working iteratively, along the process of interviewing new themes derived from the interviews and the topic guide has been adjusted. The analysis was performed independently by two researchers and the program ATLAS.ti was used for the coding process. Any disagreement on the data, categories or themes between the two researchers was resolved by discussion. The final analyses was discussed with all authors.

Ethical considerations

The healthcare professionals gave permission via recorded consent to use their experiences and interview data to evaluate the AGE-ICU tool. To respect anonymity of the healthcare professionals, transcripts were directly anonymized and audio records were deleted after transcription. This research has been approved by the local research committee of St. Antonius hospital, Nieuwegein (P21.005).

Results

Five main themes were derived from the data for the AGE-ICU evaluation and five main themes for the conversation about treatment limitation. These themes and its subthemes will be further explained below and are supported by quotes from the healthcare professionals.

The healthcare professionals were first asked about the conversation about treatment limitation in general, followed by questions about the AGE-ICU and the evaluation of the contribution to the conversations about treatment limitation.

\section{Conversation about treatment limitation}

Figure 1: Conversation about treatment limitation themes and subthemes

Careful consideration

Healthcare professionals indicated that they have to consider all options and avoid medically irresponsible treatments. "It is important to estimate in advance, before you admit someone to the intensive care, firstly that you consider the chances of success and secondly whether you think it will add something to the patient's quality of life in the future" [Interview 1]. It is important that patients are aware of their situation and that expectations are managed. Healthcare professionals mentioned that some patients are afraid for what happens when they decide not to be treated in the ICU.Giving perspective is important and talking about it can help patients and their families. "People are sometimes afraid that with pneumonia they will die of dyspnea. It is good that when family comes along, you can explain that if it is not going well and the patient becomes short of breath, you can provide palliative care" [Interview 1].

The conversation is a part of the job

Some healthcare professionals argued that it is important to have these conversations and that they can be satisfactory. They see it as achallenge to come to a shared decision with the patient. If the conversation comes to a good end it can also be satisfying. It all has to do with the practicability of the conversations and how you communicate with the patient. "In these conversations it really depends on what the tone is of what you say and how you present things, communication and nuance are very important" [Interview 8]. They mention that the conversation is part of the job. "I have no qualms about having these kinds of conversations. I think it is something that belongs to my profession and it is a beautiful challenge to do it well" [Interview 8].

The burden of the conversation

The healthcare professionals mentioned that the conversations can be complicated. It can be complicated because sometimes there is adiscrepancy or dissension of ideas between the doctor and the patient. "In 
some situations, I have the idea that what you do is never actually good. Because sometimes, when a patient dies, that is not what the family wanted. But also, when someone survives the ICU but with a low quality of life, it is also not what the family wanted" [Interview 7]. The healthcare professionals also indicated that there are situations where there is a disagreement with the patient about the treatment limitation. In some cases this leads to a certaincommotion. "Yes, I have also been accused of not wanting to treat someone anymore or something... helping someone more towards death..." [Interview 5]. Cultural difference is also a subject that has been mentioned by the healthcare professionals as being difficult sometimes. Some families, because of their religious beliefs, do not want to give up treatment. Instead they want to do everything that is possible to prevent the patient from dying even though in some cases this is medically irresponsible in the professional's eyes. This can lead to differences in opinion and unpleasant situations. "There are also transcultural problems, that we as doctors think that it is better if the patient doesn't go to the intensive care, but the family is pushing us to do so, regardless of the situation of the patient. These are difficult situations..." [Interview 7].Healthcare professionals also indicated that the conversations will be normalized for patients as long as they are consequently performed.

"So eventually everyone will get used to the conversations about treatment limitation" [Interview 9].

Scheduling conversation

In the conversation with the healthcare professionals about the best moment for the conversation it appears that there is never a right moment to talk about the treatment limitations. "Many people don't want that [talking about treatment limitations], they don't want to think about it at all" [Interview 6]. Healthcare professionals indicated that they have to perform the conversations on the emergency room (ER), however they mentioned that this is not a suitable place. Besides this, it is often difficult to have the conversation at this moment due to lack of time "I understand, from the hospital's perspective, that we have to perform these conversations on the ER. But the ER can be hectic sometimes, it is not a suitable moment" [Interview 5]. Some argue that the best moment is before someone comes to the ER, which could be at an appointment at the clinic or with the general practitioner. But in every situation, you overwhelm patients with these questions. Advance care planningis often mentioned by the healthcare professionals as important, so patients are prepared. "It is important that someone can talk and think about treatment limitations before the acute moment. So ideally, I would say advance care planning, beforehand" [Interview 6].

Acquiring skills to perform the conversation

The healthcare professionals initiate that the conversation about treatment limitation is a skill that you do not learn beforehand but improves by getting more experience. "When I just started as a doctor I used to only have the conversations about treatment limitations with patients I thought it was necessary, but I don't do that anymore. Now I talk about treatment limitations with all of my patients" [Interview 4]. The residents mentioned that they often struggle with these conversations and that it is very important to involve the supervisor to get guidance whenever the situation is difficult. "In general, I just started working and this [conversation about treatment limitation] is something you don't learn in school or during the internships, personally I find the conversations about treatment limitations often difficult and hard" [Interview 5]. The residents mentioned that sometimes it is helpful to use the opinion of experts like the geriatrician or the intensivist.

\section{AGE-ICU Evaluation}

Figure 2. AGE ICU themes and subthemes

Considered and comprehensible overview/start of conversation

Healthcare professionals mentioned that the AGE-ICU is convenient to give an overview of the patient's comorbidities and health status. It is mentioned by the professionals that by using the tool it is easier to explain the options to consider to patients. It can clarify the health status of the patient and it can be shown to the patient during the conversation. The tool contributes to an improved and substantiated conversation. It shows the full picture of the patient and helps to structure thoughts and observations. 
"I think that these kinds of decisions need to be substantiated in a structured way, and the tool is helpful for that I think" [Interview 1].

Confirmation of own assessment

In relation to the theme above, according to healthcare professionals, the AGE-ICU shows the full picture of the patient, which contributes to a comprehensible overview. "The AGE-ICU gives you a lot of arguments, and the colored bars give you a clear overview of the status of the patient [Interview 6]. Besides this, the tool canobjectify the feelings that a healthcare professional has about a patient. Healthcare professionals often use the AGE-ICU in cases of doubt. Respondents mentioned that in some cases there is no doubt about the treatment the AGE-ICU is not necessary. The AGE-ICU is often used to objectify the doctor's assessment in cases where it is not clear or difficult to estimate the chances of survival, "It is often a feeling, what you have with a patient on which you base your decision to not admit someone to the IC. By using the $A G E-I C U$ you can make that illustrative. You can say for yourself, hey this is right indeed. Because if we put this patient in the AGE-ICU screening, this patient will eventually come out bad. This confirms your feelings" [Interview 4].

Every decision is context and person dependent

As described above, healthcare professionals argue that in some cases it is very clear whether or not a patient should be admitted to the ICU. "But for the group in between, that grey area, it can be very useful to reach a decision" [Interview 3]. In these situations, the healthcare professionals mentioned that the AGE-ICU can contribute to give an overview of the aspects in consideration. It is important to estimate the chances of ICU survival based on the patients functioning and quality of life before the acute moment. Healthcare professionals indicate that the decision is fully context and person depended. "So, for every patient it is actually a separate interpretation" [Interview 1]. It is also mentioned that the tool works both for situations where you underestimate someone's chances and where you overestimate the chances of survival. "But also the other way around, when someone from a certain age is very vital and the AGE-ICU only shows green bars, than I would think yes.. this person chances for surviving the ICU are higher than I first estimated" [Interview 5].

\section{Contributes to considered decision}

The healthcare professionals indicated that the AGE-ICU is a tool that can contribute to decision making. However, they argue that the tool is not decisive because every situation needs an individual judgement. "It will always depend on how people think about the situation. And so, partly it is objectifiable, but partly it is also subjective" [Interview 9]. The healthcare professionals also mention that with the tool the patient and the family are more involved in decision-making and therefore the tool contributes toshared-decision making. "Then you can sit down together and make the decision together" [Interview 4].

Tool not needed because of own expertise

Not all healthcare professionals use the AGE-ICU consistently. Some find the tool very helpful to support in the decision. Other healthcare professionals indicated that the tool is not neededand that their own expertise is sufficient to estimate the chances of survival. "I don't need that tool to make the right decision" [Interview 3]. Many healthcare professionals describeintuition being the base for ICU admission and estimation of someone's chances. On the other hand, some respondents argued that it would be best to fill in the AGE-ICU for every patient. "But I think that the people who don't look fit, for them you fill in the tool. But it would be very good to use the tool for the people that do look fit. But now you often see that the tool is only used for people where you think at first sight... hmm this patient does not look good, that feeling..." [Interview 2].

The covid-19 crisis is distinguished from other diseases due to emotional and physical isolation. Healthcare professionals initiated that the conversations about treatment limitations are also difficult for the patients and their families. It is more difficult for the families to fully trust the professionals when family is not always allowed to be around the patient and apprehend what is happening. The healthcare professionals 
also mentioned that many patients are afraid and have the feeling that they are given up on. But because of all the news about COVID-19, people know better what to expect from the disease and ICU admission. "And that's where corona helped" [Interview 6].Discussion

This study has answered the main research question: "How do healthcare professionals experience conversations about treatment limitation with older hospitalized COVID-19 patients and how does a tool for risk-assessment contribute to these conversations?" The main findings suggest that the AGE-ICU seems to be a helpful tool which structures thoughts and gives a good overview of the patients functioning and health status. It can be assumed that the AGE-ICU is an easy-to-use tool, that assists healthcare professionals in the triage and risk assessment of older patients infected with COVID-19. The conversations can be difficult and specifically in situations with cultural differences.

The right moment for the conversation is a difficult consideration. Patients will always be overwhelmed wherever and whenever the conversation will be held. Patients that are infected with the coronavirus can deteriorate very quickly ${ }^{18}$, therefore it would be best that the conversation is performed before this occurs. It is most conducive if patients have thought and talked about treatment limitations before the acute moment.

According to healthcare professionals advance care planning is important, to prepare patients for this moment ${ }^{19}$. If patients know that they no longer want treatment, this can also save unnecessary treatment or transportation to the hospital. However, in reality many patients have not thought about it yet, because advance care planning is not yet standardized ${ }^{20}$. Therefore, it is even more important that healthcare professionals are provided with tools, such as the AGE-ICU, to contribute to the conversation at the moment a treatment decision has to be made.

The healthcare professionals indicated that for some patients it is very clear if ICU treatment could be beneficial or not, consequently the AGE-ICU is most often used for patients in the grey area. However, how these cases of doubt are identified is unclear and often based on the healthcare professional's subjective assessment. According to them, the AGE-ICU is a suitable tool to objectify their assessment in cases where it is not clear or difficult to estimate the chances of survival.

Whereas many healthcare professionals find the AGE-ICU valuable, some indicate that their own expertise is sufficient for the assessment of older COVID-19 patients. Perhaps, the most serious disadvantage of not using a tool is the in-transparency for the patients because the decision is subjective. Recent literature investigated tools for advance care planning and goals of care discussions, they concluded that consensus about using tools for these discussions does not exist among healthcare professionals ${ }^{19}$. This is in line with the findings of this study. The AGE-ICU is a tool that could be used to objectify the healthcare professional's assessment regarding frailty, resulting in a better decision. Additionally, the tool is helpful to explain a decision to the patient because by using the tool it is more insightful for patients.

The AGE-ICU can be used as a visualizer for the patient, they can get insight in their current functioning and health status which can be very clarifying. Also, patients are more involved in the decision. By applying shared-decision making the autonomy of patients is respected and another advantage is that shared-decision making is associated with higher patient satisfaction ${ }^{13}$. This research shows that in times healthcare professionals find the conversation difficult to perform. Especially in situations with cultural differences in opinion, which sometimes leads to unpleasant discussions. On the other hand, the healthcare professionals mentioned that when these situations occur, it makes no difference if the person that performs the conversation is an experienced doctor or not. By using the AGE-ICU the healthcare professionals' assessment is objectifiable and doctors, experienced in these conversations or not, can develop their skills. Additionally, by using the tool the considerations can be transparent for the patient. Even more important, is the tone of communication and sensitivity in the way the news is delivered ${ }^{21}$. Myers et al (2018) concluded that communication skills development is required to perform effective conversations about goals of care and advance care planning education $^{19}$.

Hubbard et al. (2020) concluded that a screening tool needs to be a part of the healthcare professional's assessment and that the severity of the acute illness, the likelihood of treatment success and the degree 
of frailty needs to be considered ${ }^{5}$. The AGE-ICU screening tool contributes to objectify the professionals' assessment, shows chances of success and includes frailty. Every patient needs an individual approach and therefore the AGE-ICU only makes the patients' health status and functioning visual, but does not conclude anything.

A limitation of this study is the risk for subjectivity from the researchers. The data is interpreted as open as possible, nonetheless preformed prejudices and the lens that the researchers build through experiences can be a risk for subjectivity. The analysis is performed by two researchers to minimize the risk for subjectivity.

Conclusion

The AGE-ICU is a tool that contributes to conversations about treatment limitation, which are often experienced as difficult by health care professionals. It gives a clear overview of risk factors for adverse outcome in older COVID-19 patients and offers insights through a user-friendly interface. The AGE-ICU helps to objectify the patients' health status and functioning and helps the healthcare professionals with the difficult conversation with the patient and their family. Additionally, the AGE-ICU contributes to shared-decision making because it helps patients to understand the suggested decision. Given the fact that the ER can be hectic sometimes, it is necessary to continue to develop the tool, so that it is also user-friendly in hectic times on the ER. A possible area of future research would be to investigate the patient's perspective on the conversations about treatment limitation and the value of the AGE-ICU in this conversation.

\section{References}

1. Wiersinga WJ, Rhodes A, Cheng AC, Peacock SJ, Prescott HC. Pathophysiology, Transmission, Diagnosis, and Treatment of Coronavirus Disease 2019 (COVID-19): A Review. JAMA. 2020;324(8):782-93.

2. Coronavirus disease (COVID-19) pandemic [Internet]. WHO. 2021. Available from:https://www.who.int/emergencies/diseases/novel-coronavirus-2019 .

3. Maltese G, Corsonello A, Di Rosa M, Soraci L, Vitale C, Corica F, et al. Frailty and COVID-19: A Systematic Scoping Review. J Clin Med. 2020;9(7).

4. Antommaria AHM, Gibb TS, McGuire AL, Wolpe PR, Wynia MK, Applewhite MK, et al. Ventilator Triage Policies During the COVID-19 Pandemic at U.S. Hospitals Associated With Members of the Association of Bioethics Program Directors. Ann Intern Med. 2020;173(3):188-94.

5. Hubbard RE, Maier AB, Hilmer SN, Naganathan V, Etherton-Beer C, Rockwood K. Frailty in the face of COVID-19. Age Ageing. 2020;49(4):499-500.

6. Ballantyne A, Rogers WA, Entwistle V, Towns C. Revisiting the equity debate in COVID-19: ICU is no panacea. J Med Ethics. 2020;46(10):641-5.

7. De Smet R, Mellaerts B, Vandewinckele H, Lybeert P, Frans E, Ombelet S, et al. Frailty and Mortality in Hospitalized Older Adults With COVID-19: Retrospective Observational Study. J Am Med Dir Assoc. 2020;21(7):928-32 e1.

8. de Jonge E, Mooijaard SP. Framework to decide on withholding intensive care in older patients. Netherlands Journal of Critical Care. 2019;27(4):150-4.

9. Muscedere J, Waters B, Varambally A, Bagshaw SM, Boyd JG, Maslove D, et al. The impact of frailty on intensive care unit outcomes: a systematic review and meta-analysis. Intensive Care Med. 2017;43(8):110522 .

10. Vincent JL, Creteur J. Ethical aspects of the COVID-19 crisis: How to deal with an overwhelming shortage of acute beds. Eur Heart J Acute Cardiovasc Care. 2020;9(3):248-52.

11. Griffiths F, Svantesson M, Bassford C, Dale J, Blake C, McCreedy A, et al. Decision-making around admission to intensive care in the UK pre-COVID-19: a multicentre ethnographic study. Anaesthesia. 2020. 
12. Walczak A, Butow PN, Davidson PM, Bellemore FA, Tattersall MH, Clayton JM, et al. Patient perspectives regarding communication about prognosis and end-of-life issues: how can it be optimised? Patient Educ Couns. 2013;90(3):307-14.

13. Berghuis L. Physician-related Factors concerning Treatment Limitations A qualitative study. 2019.

14. Myrstad M, Ihle-Hansen H, Tveita AA, Andersen EL, Nygard S, Tveit A, et al. National Early Warning Score 2 (NEWS2) on admission predicts severe disease and in-hospital mortality from Covid-19 - a prospective cohort study. Scand J Trauma Resusc Emerg Med. 2020;28(1):66.

15. Pel EL, van Wijngaarden E, van Dongen EPA, Noordzij PG. Anesthesia Geriatric Evaluation (AGE): A Care-Ethical Perspective of a Multi-Disciplinary Approach for Tailored Preoperative Interventions. Gerontol Geriatr Med. 2019;5:2333721419876126.

16. Green J, Thorogood N. Qualitative Methods for Health Research: SAGE Publications; 2018.

17. Gale NK, Heath G, Cameron E, Rashid S, Redwood S. Using the framework method for the analysis of qualitative data in multi-disciplinary health research. BMC Med Res Methodol. 2013;13:117.

18. Perrotta F, Corbi G, Mazzeo G, Boccia M, Aronne L, D'Agnano V, et al. COVID-19 and the elderly: insights into pathogenesis and clinical decision-making. Aging Clin Exp Res. 2020;32(8):1599-608.

19. Myers J, Cosby R, Gzik D, Harle I, Harrold D, Incardona N, et al. Provider Tools for Advance Care Planning and Goals of Care Discussions: A Systematic Review. Am J Hosp Palliat Care. 2018;35(8):1123-32.

20. Bradshaw A, Dunleavy L, Walshe C, Preston N, Cripps RL, Hocaoglu M, et al. Understanding and addressing challenges for advance care planning in the COVID-19 pandemic: An analysis of the UK CovPall survey data from specialist palliative care services. Palliat Med. 2021:2692163211017387.

21. Cardona M, Anstey M, Lewis ET, Shanmugam S, Hillman K, Psirides A. Appropriateness of intensive care treatments near the end of life during the COVID-19 pandemic. Breathe (Sheff). 2020;16(2):200062.

Acknowledgments

The authors express their gratitude to the healthcare professionals of St. Antonius hospital Nieuwegein who participated in the study. This research did nog receive any funding in the public, commercial, or non-profit sectors.

Conflict of interest statements

The authors declare no conflict of interest.

Figure Legends

Figure 1: Conversation about treatment limitation themes and subthemes. This figure depicts the different themes and subthemes derived from the data evaluating the experiences of healthcare professionals regarding conversations about treatment limitation.

Figure 2. AGE ICU themes and subthemes. This figure depicts the different themes and subthemes derived from the data evaluating the AGE ICU tool.

Appendix

Figure 3: Conversation about treatment limitation; Codes, Subthemes and Themes. This figure depicts the different codes derived from the data evaluating the experiences of healthcare professionals regarding conversations about treatment limitation. The figure shows the subthemes and themes conjugated from the codes.

Figure 4: AGE ICU evaluation; Codes, Subthemes and Themes. This figure depicts the different codes, subthemes and themes derived from the data evaluating the AGE ICU tool. The figure shows the subthemes and themes conjugated from the codes. 


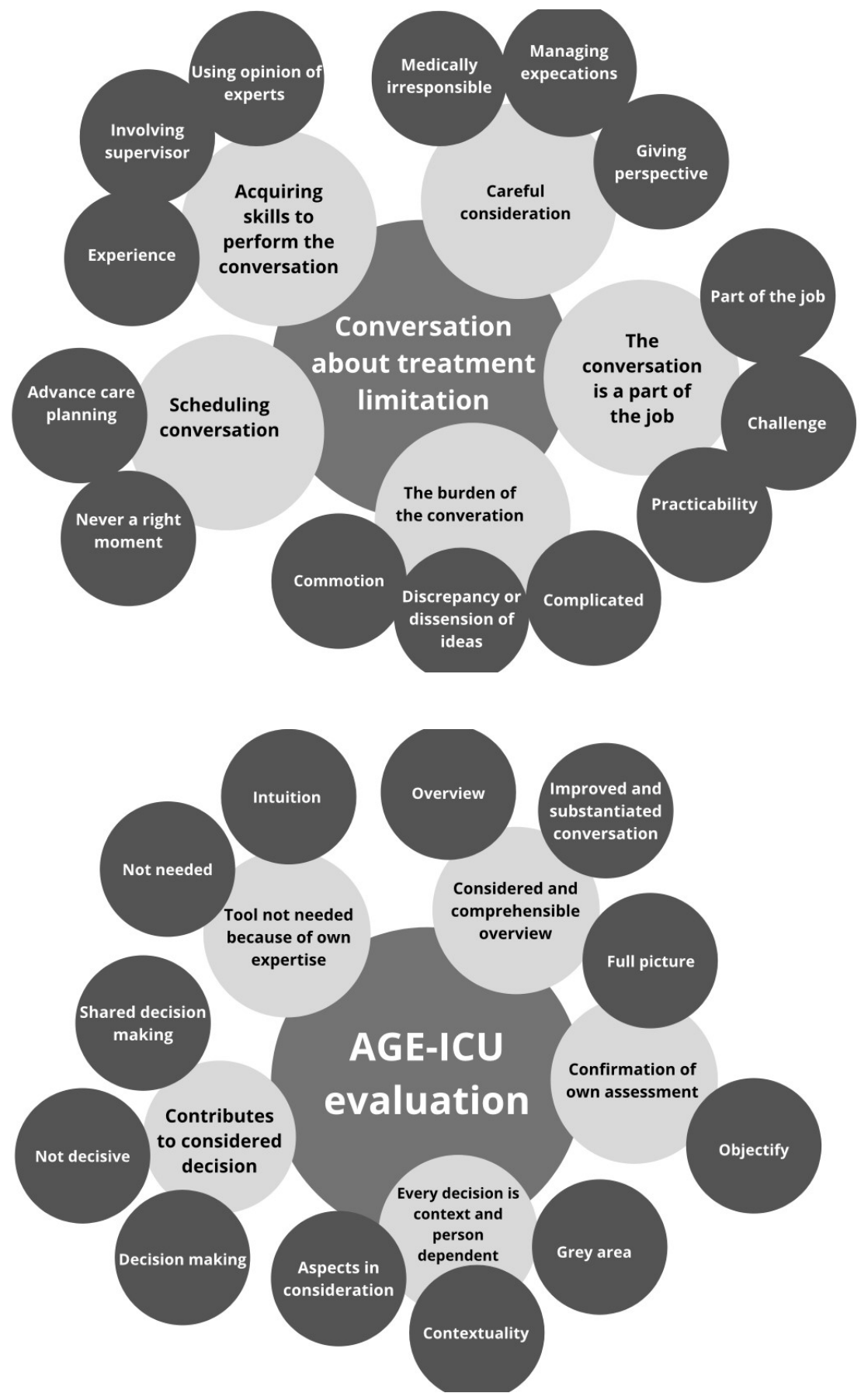

\section{Hosted file}

Appendix-Figure 3.pdf available at https://authorea.com/users/460494/articles/556479discussing-treatment-limitations-in-frail-older-covid-19-patients-a-framework-analysis

Hosted file

Appendix - Figure 4.pdf available at https://authorea.com/users/460494/articles/556479discussing-treatment-limitations-in-frail-older-covid-19-patients-a-framework-analysis 